\title{
Mental Health and Substance Abuse
}

\section{The Effects of Health Sector Market Factors and Vulnerable Group Membership on Access to Alcohol, Drug, and Mental Health Care}

\author{
Susan E. Stockdale, Lingqi Tang, Lily Zhang, Thomas R. Belin, \\ and Kenneth B. Wells
}

Objective. This study adapts Andersen's Behavioral Model to determine if health sector market conditions affect vulnerable subgroups' use of alcohol, drug, and mental health services (ADM) differently than the general population, focusing specifically on community-level predisposing and enabling characteristics.

Data Sources. Wave 2 data (2000-2001) from the Health Care for Communities study, supplemented with cases from wave 1 (1997-1998), were merged with area characteristics taken from Census, Area Resource File (ARF), and other data sources.

Study Design. The study used four-level hierarchical logistic regression to examine access to ADM care from any provider and specialty ADM access. Interactions between community-level predisposing and enabling vulnerability characteristics with individual race/ethnicity, age, income category, and insurance type were explored.

Principal Findings. Nonwhites, the poor, uninsured, and elderly had lower likelihoods of service use, but interactions between race/ethnicity, income, age and insurance status with community-level vulnerability factors were not statistically significant for any service use. For ADM specialty care, those with Medicare, Medicaid, private fully managed, and private partially managed insurance, the likelihood of utilization was higher in areas with higher HMO penetration. However, for those with other insurance or no insurance plan, the likelihood of utilization was lower in areas with higher HMO penetration.

Conclusions. Community-level enabling factors explain part of the effect of disadvantaged status but, with the exception of the effect of HMO penetration on the relationship between insurance and specialty care use, do not modify any of the residual individual-level effects of disadvantage. Interventions targeting both structural and individual levels may be necessary to address the problem of health disparities. More research with longitudinal data is necessary to sort out the causal direction of social context and ADM access outcomes, and whether policy interventions to change health sector market conditions can shift ADM treatment utilization. 
Key Words. Mental health, substance abuse, behavioral model, access/utilization, service availability

Access to care for alcohol, drug, and mental health (ADM) conditions is the outcome of a complex dynamic involving demand and supply factors at both the system and individual levels. While some research posits that access for everyone in the community may be compromised as poverty and population racial/ethnic distribution lead to reductions in community-wide service availability, other research suggests that certain vulnerable subgroups (nonwhites, the elderly, the poor, and the uninsured) will be disproportionately, and negatively, affected (IOM 2003). Although a number of studies have examined individual pieces of this dynamic, few if any have explored at a national level the combined effects of community-level socioeconomic disadvantage (i.e., poverty and racial/ethnic distribution), safety net development, service availability, and individual risk factors on access to ADM care. Using a large national sample, this study tests a conceptual model of access based on the expansion of Andersen's Behavioral Model for vulnerable populations (Gelberg, Andersen, and Leake 2000) and explores whether certain health sector market conditions compound disparities for vulnerable subgroups.

\section{CONCEPTUAL MODEL}

Andersen's original behavioral model, developed in the 1960s, posited that health services use is a function of predisposing, enabling, and need factors (Andersen 1995). A more recent expansion of the model to explain utilization and outcomes for vulnerable populations distinguishes between traditional and vulnerable domains within predisposing, enabling, and need factors (Gelberg, Andersen, and Leake 2000). Based on the literature about problems affecting utilization of care by the homeless, Andersen and colleagues add new "vulnerability" predictors. More recent iterations of the behavioral model also point to the importance of including enabling and predisposing factors, as well as a vulnerability domain, at the community level; such a model has not been

Address correspondence to Susan E. Stockdale, Ph.D., UCLA Semel Institute Health Services Research Center, 10920 Wilshire Blvd., Ste 300 Los Angeles, CA 90024. Lingqi Tang, Ph.D., Lily Zhang, M.A., and Kenneth B. Wells, M.D., M.P.H., are with the UCLA Semel Institute Health Services Research Center, Los Angeles, CA. Thomas R. Belin, Ph.D., is with the Department of Biostatistics, UCLA School of Public Health, Los Angeles, CA. 
developed for ADM services use. In addition, the possibility of cross-level interactions between community and individual levels has not been well conceptualized within Andersen's model. By including cross-level interactions between community-level vulnerability factors and individual-level characteristics, we can determine if some vulnerable subgroups are more negatively impacted by unfavorable health sector market conditions.

To develop a framework to explain ADM treatment utilization with a particular focus on the poor, uninsured, elderly, and nonwhites, we follow Gelberg, Andersen, and Leake's (2000) approach and turn to literature on ADM service utilization to identify important vulnerability factors at the community level. The IOM and others argue that community socioeconomic disadvantage, particularly poverty (Chow, Jaffee, and Snowden 2003) and percent nonwhite population (Chandra and Skinner 2003), creates unfavorable health sector market conditions that lead to reduced access to services. Our model incorporates these community-level social structure and demographic variables as predisposing vulnerability factors, along with traditional predisposing factors such as individual-level race/ethnicity, education, employment, and marital status.

Andersen (1995) has suggested that broadening the scope of community enabling factors beyond traditional service availability measures (physicians per capita and number of facilities) to include more information about the health services sector would improve the ability of the behavioral model to explain service use. While greater service availability at the community level (i.e., psychiatrists per capita, MDs per capita, inpatient psychiatric units) has been associated with increased access and use of mental health services (Hendryx, Urdaneta, and Borders 1995), two additional health sector market factors identified in the literature may be particularly important for explaining ADM service use by vulnerable subgroups: community uninsurance/safety net development and $\mathrm{HMO}$ presence. As suggested by the literature discussed below, in addition to traditional service availability measures, our conceptual model expands the community-level enabling domain to include the percent of the population uninsured and HMO market penetration as additional vulnerability factors.

Studies of access to mental health care and treatment of mental disorders show that people living in communities with a better developed safety net for the uninsured (more public health collaborations with state and local community agencies, higher levels of public insurance coverage) have greater access to mental health care (Hendryx and Ahern 2001). Conversely, high community uninsurance rates may overburden existing services with uncompensated care, forcing providers, hospitals, and clinics to limit access to services (IOM 2003). This shortage of charity care is 
particularly likely to decrease access to care for low-income uninsured persons (Cunningham 1999).

Evidence of the effects of HMO market penetration on access to care is less clear. Although several studies demonstrate better initial access to health and mental health services under managed care, including behavioral health "carve-outs" (Sturm 1997; Goldman, McCulloch, and Sturm 1998; Grazier and Eselius 1998; Grazier et al. 1999; Gresenz, Stockdale, and Wells 2000; Hendryx et al. 2002), some of these also indicate that intensity or volume of services decreases. One study found that although the introduction of managed care did not affect access to mental health specialty care, patients in more managed plans were less likely to receive a referral to specialty mental health care (Grembowski et al. 2002), and another study conducted in Ohio found worse access to health care in areas with higher HMO market penetration (Litaker and Cebul 2003). In addition, a few studies have raised concerns about strategies used by managed care to reduce costs, such as restricting number of outpatient visits and specialty care visits, arguing that lower costs per plan member may indicate restricted access to services (Weissman et al. 2000; Wilk et al. 2005).

Some evidence also indicates that the growth of managed care may result in decreased access to services for nonwhites, the poor, the elderly, and the uninsured. In particular, cost-sharing mechanisms may disproportionately affect nonwhites and the poor, who may forego care altogether if they cannot cover their share of the cost or the high insurance premiums (Rice 2003). Although Fiscella and Franks (2005) found no racial/ethnic disparities in care associated with HMO participation, one recent study indicates that nonwhites are less likely than whites to receive antidepressant medication for newly diagnosed episodes of depression and were less likely to receive posthospitalization followup (Virnig et al. 2004). Likewise, some evidence (Feinson and Popper 1995; Grazier and Eselius 1999) indicates that fragmentation of care associated with managed care, as well as other organizational and structural factors, may represent a barrier to access for vulnerable groups such as the elderly, who are more likely than younger people to experience depression but are less likely to use ADM services (Krause 1999; Karlin and Norris 2005). Other research has shown that low-income uninsured persons have worse access to medical care in communities with high levels of uninsurance and high levels of Medicaid managed care penetration (Cunningham and Kemper 1998; Cunningham 1999), and increases in $\mathrm{HMO}$ market penetration have been associated with decreases in uncompensated care (Thorpe, Seiber, and Florence 2001).

In the analyses below, we explore the effect of community level "traditional" and "vulnerable" predisposing and enabling domain factors on 
ADM service use. Based on the literature discussed above, we hypothesize that lower service use is associated with lower rates of service availability, a traditional predisposing factor identified by Andersen and colleagues. As suggested by our extension of the behavioral model for vulnerable populations and supported by the literature, we further hypothesize that the effect of the additional community-level predisposing and enabling vulnerability factors will be a function of race/ethnicity, age, income, and insurance status. We expect that higher community poverty, percent nonwhite population, and percent uninsured will result in less service use by nonwhites, the poor, the uninsured, and the elderly. However, given the mixed findings with regard to managed care, we are unsure whether higher HMO market penetration will result in better or worse access, or no effect, for vulnerable groups.

\section{METHODS}

\section{Data}

We used cross-sectional data from wave 2 of the telephone survey from Healthcare for Communities (HCC), supplemented with cases from wave 1 of HCC. The first wave of HCC (1997-1998) used a sample of 14,985 drawn from a pool of 30,375 respondents to the first wave of the Community Tracking Study (CTS, 1996-1997); a total of 9,585 completed interviews were obtained (64 percent response rate) (Sturm et al. 1999). HCC oversampled individuals from the CTS with low incomes, ADM services use in the past year, and high psychological distress, in order to increase the precision and power of estimates for ADM need, access, and treatment. All HCC wave 1 participants were included in the wave 2 sampling frame, and an additional sample of 10,500 individuals was drawn from 39,504 respondents to wave 2 of CTS (1998-1999). A total of 12,158 respondents completed HCC wave 2 (fielded during 2000-2001) for a response rate of 60.5 percent. In order to increase the sample size for this analysis, in addition to the wave 2 sample we included wave 1 data for 2,926 HCC participants who were in the sampling frame for wave 2 but did not complete the wave 2 survey.

We merged the individual-level survey data with community-level data from various sources. Because our measures for HMO penetration and percent uninsured are based on data for Metropolitan Statistical Areas (MSA) and were extrapolated to non-MSA counties (Bureau of Health Professions 1999; Bureau of Labor Statistics and Bureau of the Census 2000), we excluded nonMSA counties from the analyses. With the exception of two variables, the 
community-level data (described in more detail below) were measured at the county level. Inpatient/outpatient facilities and percent nonwhite were measured at the zip code and Census tract levels, respectively. After eliminating cases with missing data and the non-MSAs, the final sample for analysis comprised 11,137 individuals.

\section{Measures}

Outcomes. We constructed an indicator for access to any ADM services (past 12 months) through either primary care or specialty provider that included some form of assessment, monitoring, or treatment. For primary care, this was defined as any visit during which the clinician suggested that the respondent cut down on alcohol or drugs, referred the respondent to specialty ADM care, suggested medication for a substance abuse or mental health problem, counseled the respondent for $5 \mathrm{~min}$ or more about an ADM problem, or asked about emotional or mental health problems, or alcohol or illicit substance use. A specialty care visit was defined as visiting a mental health or substance abuse provider, such as a psychiatrist, psychologist, social worker, or psychiatric nurse; having a hospital admission or emergency room visit in the last 12 months for an ADM problem; or attending an inpatient or outpatient alcohol or drug program.

In addition to any ADM services, we modeled the indicator for use of specialty care services separately.

Predisposing Characteristics. In our models to predict use of ADM services, we included all the "traditional" predisposing characteristics, except health beliefs (because these were not measured in the HCC surveys). These included race/ethnicity (white, black, Hispanic, other), education (less than high school, high school graduate, college graduate), gender, married or living with a partner as married, employment status (unemployed versus employed), and age category (18-34, 35-64, 65 years or older).

Additional vulnerability characteristics included in Andersen and colleagues' model to understand service use and outcomes of the homeless included prison history, victimization, neighborhood of residence, history of homelessness, type of shelter, and history of mental illness and substance abuse disorders and hospitalizations (Gelberg, Andersen, and Leake 2000). Based on previous research demonstrating the importance of area poverty and racial/ethnic population distribution, we included measures of these community-level variables, obtained from the 2000 U.S. Census (U.S. Census 2000), as predisposing vulnerability factors. Poverty was measured 
continuously as percent of all people in poverty at the county level. We used a continuous measure of percent nonwhite population, measured at the Census tract level, and constructed from Census 2000 population counts by race and by Hispanic ethnicity.

Of the individual-level vulnerability characteristics identified by Andersen and colleagues, those also measured in the HCC surveys and that seemed most applicable for our vulnerable subgroups included exposure to violence (as a measure of life stress) and indicators for the following probable 12-month disorders: major depressive disorder, dysthymic disorder, generalized anxiety, panic disorders, lifetime psychosis, lifetime mania and alcohol abuse or illicit drug use. With the exception of the measures for psychosis and mania, these measures are widely used and have been found to have excellent reliability and validity (WHO 1990, 1992, 1997; Burnam and Young 1996; Kessler et al. 1998). We also included an additional measure of count of chronic conditions (of 17 possible). In addition, the hierarchical logistic regression technique that we used accounts for neighborhood of residence by modeling random intercepts for Census tracts and counties.

Enabling Characteristics. Of the traditional enabling characteristics identified by Andersen, we included type of insurance (no insurance, Medicaid, Medicare, private plan with no managed care, private plan with partial managed care, and private plan with fully managed care), total family income (collapsed into quartiles of the empirical distribution) and functional social support (measured continuously, average of six items measured on a six-point Likert scale) (Sherbourne and Stewart 1991). HCC did not measure (and we did not include) an indicator for having a regular source of care. To measure community level enabling factors, from the Area Resources File we constructed a continuous measure at the county level of psychiatrists (patient care) per 100,000 population for 1998. We also included the availability of inpatient and outpatient ADM care facilities, derived from ZIP Code Business Patterns data from the U.S. Census Bureau and U.S. Department of Commerce (1999) and calculated as the sum of inpatient and outpatient facilities within zip code.

As vulnerability enabling factors, we included individual-level family wealth to represent the resources available to pay for ADM services. At the community level, other possible vulnerability factors identified by Andersen and colleagues include social service availability and crime rates (Gelberg, Andersen, and Leake 2000). For the vulnerable subgroups we focused on here, 
the availability of charity care for the uninsured, the indigent, and those who cannot afford care is particularly important. As a proxy for the availability of charity care, we included the percent uninsured in 2000, calculated as a 3-year moving average from Current Population Survey estimates (March supplement) of the U.S. Census Bureau (Bureau of Labor Statistics and Bureau of the Census 2000) at the MSA (county) level. As an additional vulnerability enabling factor, we included a measure for HMO penetration rate for 1998, obtained from the Area Resources File (Bureau of Health Professions 1999). We did not include crime rates, because the literature does not identify community crime as predictive of service use for our vulnerable subgroups.

Need Characteristics. In addition to the indicators of disorders, described above with the other predisposing characteristics, we included an indicator of perceived need, based on responses to the questions "in the last 12 months, did you think you needed help for emotional or mental health problems, such as feeling sad, blue, anxious or nervous?" and "in the past 12 months did you think you needed help for alcohol or drug problems?" As an additional measure of need, we included the five-item Mental Health Inventory (MHI5), an index ranging from 0 to 100 where 100 represents well-being and 0 represents severe emotional distress in the prior 30 days (Berwick et al. 1991).

Table 1 contains (unweighted) descriptive statistics of the analysis sample.

\section{Analysis}

We estimated separate four-level hierarchical logistic regression models for our two outcomes (general ADM and specialty care access) using the software package MLwiN (Bryk and Raudenbush 1992; Rasbash, Steele, and Brown 2003), with individuals as level 1 units $(n=11,137)$ nested within households $(n=9,214)$, nested within Census tracts $(n=6,387)$, nested within counties $(n=348)$. The models included predictors for county, Census tract, and individual levels, as well as design variables measured at the level of households and individuals. The model for any ADM care included random slopes for county, Census tract, and household level. We included a random slope for the household level only in the model for ADM specialty care, as the model would not converge with nonnegative variance components when random slopes at all three levels were modeled. ${ }^{1}$ All continuous variables were centered at the grand mean for analysis. We also used an extended hot deck 
Table 1: Sample Characteristics $(n=11,137)$

Percent or Mean (SD)

\section{Individual characteristics}

Female

$61.32 \%$

Age

$18-35$ years

$26.64 \%$

36-64 years

$56.17 \%$

65 years or older

$17.19 \%$

Education

Did not complete high school

$11.57 \%$

Graduated high school

$58.73 \%$

College or higher degree

$29.70 \%$

Married or living as married

$62.45 \%$

Race/ethnicity

White

$75.84 \%$

Black

$11.86 \%$

Hispanic

$8.55 \%$

Other

$3.75 \%$

Family income

$<\$ 20,000$

$24.75 \%$

$\$ 20,000-\$ 39,999$

$22.30 \%$

$\$ 40,000-\$ 69,999$

$24.85 \%$

$\$ 70,000$ and above

$28.11 \%$

Family wealth (\$)

Health insurance coverage

Not insured

Medicare

$10.95 \%$

Medicaid

$11.88 \%$

Private with full manage

$7.52 \%$

Private with some manage

$32.83 \%$

$22.72 \%$

Private insurance with no management $\quad 11.22 \%$

Other insurance plan

$2.88 \%$

$36.35 \%$

Any ADM specialty care

$10.59 \%$

Social support

$4.7(1.2)$

Witnessed a beating, etc. in the past 12 months

$3.34 \%$

Any perceived need

$17.88 \%$

Generalized anxiety disorder

$5.61 \%$

Major depressive disorder

$13.54 \%$

Dysthymia disorder

$6.20 \%$

Mania

$2.67 \%$

Psychostism

$1.91 \%$

Panic disorder

Any alcohol or substance abuse

$7.74 \%$

MHI5

Number of chronic medical conditions

$1.6(1.8)$

Health sector market factors and community socioeconomic disadvantage

Inpatient/outpatient ADM care facilities

Psychiatrists per $100 \mathrm{k}$ population

$17.1(4.6)$

HMO penetration rate

34.0 (16.3)

Percent population nonwhite

$29.2(28.3)$

Percent of poverty 
multiple imputation technique that modifies the predictive mean matching method to impute missing items (Little 1988; Bell 1999). Several imputed variables were used in this analysis including total family income (with 27 percent missing), social support, indicators of disorders (mania, psychosis, depression, etc.), and variables used to construct any ADM care and specialty care (all with less than 2 percent missing). The results across five imputed data sets were combined by averaging, and standard errors were adjusted to reflect both within-imputation variability and between-imputation variability (Rubin 1987). We incorporated design variables and predictors of survey nonresponse as fixed effects to mitigate the impact of an informative sampling design (Pfeffermann and LaVange 1989).

In order to explore whether the additional community-level vulnerability factors explain low service use by nonwhites, the poor, the uninsured, and the elderly, we tested interactions between these characteristics and community poverty, percent nonwhite, percent uninsured, and HMO market penetration given any significant main effects. For example, if race/ethnicity, insurance type, and HMO penetration were significant in the main effects model, we then ran additional models to test an interaction between race/ ethnicity and HMO penetration and an interaction between insurance type and HMO penetration. The universe of interactions suggested by our conceptual framework included 16 possible interactions (4 individual-level characteristics $\times 4$ community-level vulnerability factors). In the main effects model for any ADM care, all four individual-level characteristics were statistically significant, as were percent population nonwhite and percent population uninsured. Thus for this outcome we tested models with interactions for: (1) race/ethnicity $\times$ percent nonwhite population; (2) race/ethnicity $\times$ percent population uninsured; (3) income category $\times$ percent nonwhite population; (4) income category $\times$ percent population uninsured; (5) age group $\times$ percent population nonwhite; (6) age group $\times$ percent population uninsured; (7) insurance type $\times$ percent population nonwhite; and, (8) insurance type $\times$ percent population uninsured. Similarly, based on the results of the main effects model for any specialty ADM care, we tested models with interactions for: (1) race/ethnicity $\times \mathrm{HMO}$ market penetration and (2) insurance type $\times \mathrm{HMO}$ market penetration. A significant interaction was found only for specialty care access.

To ascertain that we had sufficient power to detect significant cross-level interactions, we conducted sensitivity analyses for the interaction effects using Tukey's one-degree-of-freedom test for nonadditivity (Milliken and Graybill 1970). We used the following algorithm. For example, to test the interaction of 
race (a categorical variable with four categories) by percent nonwhite population (a continuously scaled variable, denoted as $W$ ), we first fit a main effects model including all predictors at the individual and neighborhood level. Based on the estimated beta coefficients from the main effects model, we create an index defined as the product of effects due to race and due to the percent nonwhite as

$$
f_{i j}=\left(\beta_{i}\right)\left[\beta(W) W_{j}\right]
$$

where $\beta_{i}$ is the $\beta$ coefficient for $i$ th race level ( $=0$ if the race $=$ white category), $\operatorname{beta}(W)=$ estimated beta coefficient for $W$, and $W_{j}=$ the value of percent nonwhite population at $j$ th neighborhood. We then refit the main effects model by including $f_{i j}$ as an additional covariate. The $\beta$ coefficient of $f_{i j}$ was interpreted as the 1-df Tukey's test and compared with the conditional $F$-test with $\mathrm{df}=3$. The results of the 1 - $\mathrm{df}$ tests were similar to the $F$-tests, and no new significant interactions were discovered.

To explore further the effects of neighborhood-level predisposing and enabling factors on any ADM access for vulnerable subgroups, given that none of the cross-level interactions were significant, we calculated the relative change in the odds ratios for race/ethnicity, age, income, and insurance type by comparing the results from a model with only individual-level predictors with a model that included all neighborhood and individual-level predictors. The change in the odds ratio was calculated as

$\left(\mathrm{OR}_{\text {individual characteristics only }} \mathrm{OR}_{\text {full model }}\right) /\left(\mathrm{OR}_{\text {individual characteristics only }}\right) \times 100$.

Expressed as a percentage change, this measure represents the amount of change in the odds of using any ADM services that is attributable to neighborhood characteristics.

To facilitate interpretation of cross-level interaction effects between HMO penetration rate and insurance type on any specialty ADM access, we calculated odds ratios for each insurance type at specific percentage values of HMO penetration rate. Tests of significance were directly derived from the logistic regression model parameters.

\section{RESULTS}

Table 2 contains odds ratios and confidence intervals for the effects of individual-level race/ethnicity, age, income, insurance status and the traditional and vulnerable predisposing and enabling community-level predictors on the 


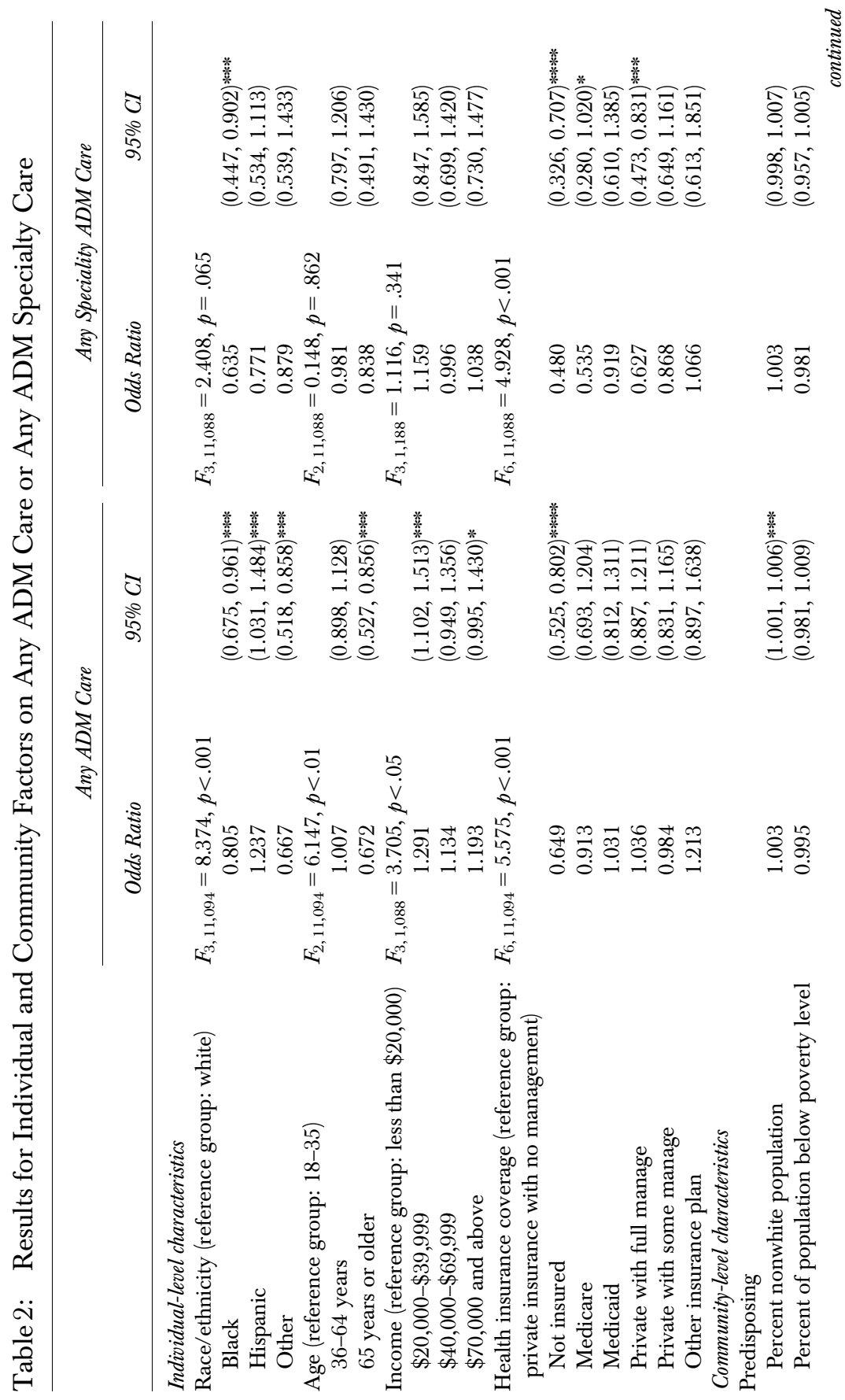




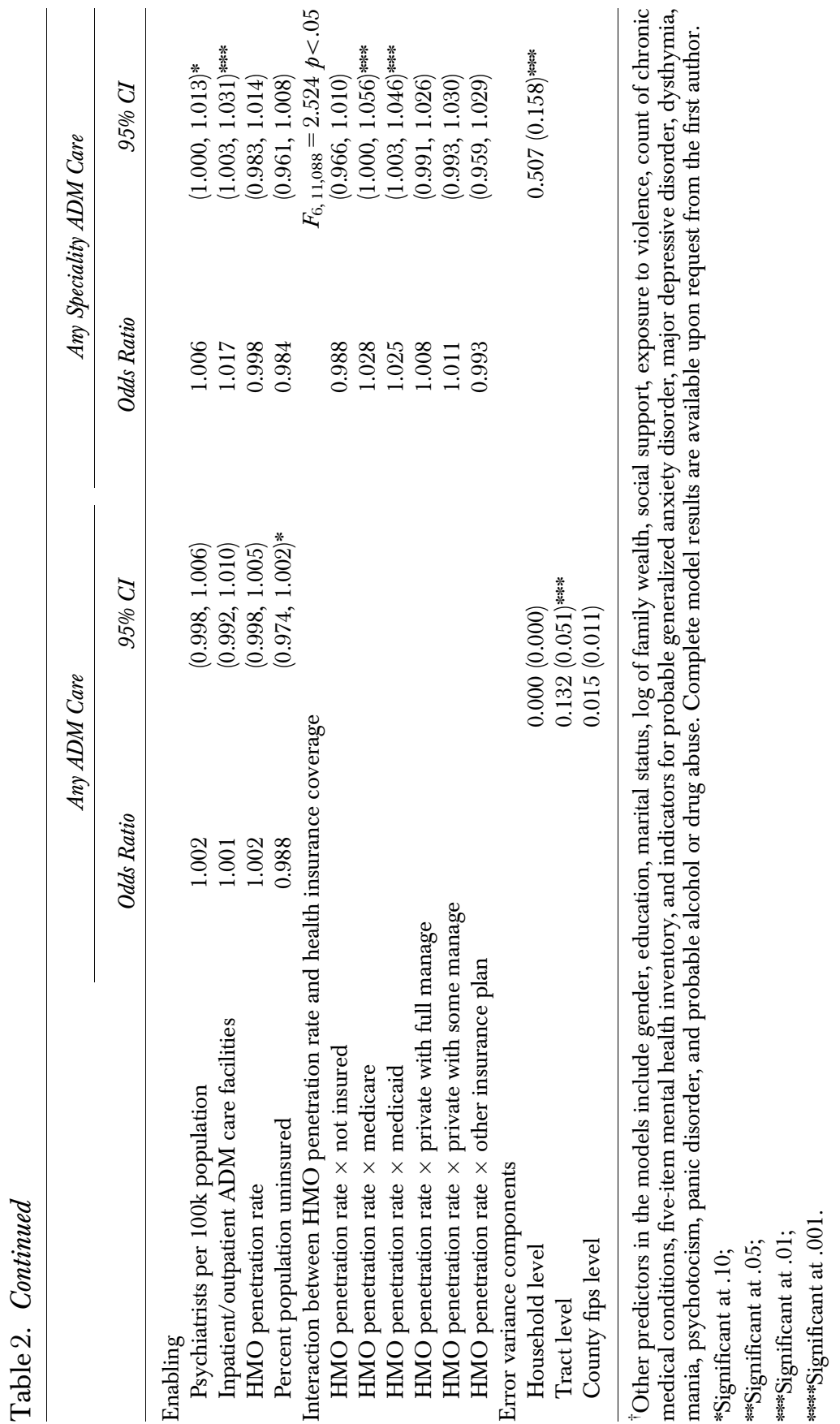


two outcomes examined here. For any ADM care, contrary to expectations higher percent nonwhite population was associated with better access to services. Percent uninsured was marginally significant $(.10>p>.05)$, and tended in the direction predicted by our hypotheses. Although, joint tests of significance for categorical variables indicated significant zero-order effects for race/ethnicity, age category, income category, and insurance type at the individual level, none of these interacted significantly $(p<.05)$ with percent nonwhite population or percent uninsured. The percentage change in the odds ratios for a model with individual characteristics only versus the full model was less than 13 percent for all vulnerable subgroups. The largest changes were for blacks ( -12.2 percent), Hispanics ( -5.6 percent), and other race/ethnicity ( -5.5 percent). These changes indicate that when community characteristics are controlled for, the likelihood of getting care for these groups decreases. Likewise, neighborhood characteristics accounted for a 3.4 percent lower likelihood of care for older people, a 2 percent lower likelihood for those with Medicare, and a less than 1 percent lower likelihood for middle-aged people, lower income $(\$ 20,000-\$ 40,000)$, higher income (more than $\$ 70,000)$, and people with Medicaid. Slight increases in likelihood of care due to neighborhood characteristics were found for middle income individuals $(0.04$ percent) and those with private fully managed insurance ( 0.58 percent), private partially managed insurance (5.24 percent), other insurance ( 0.83 percent), and no insurance ( 0.27 percent).

In addition, we found that lower general ADM service use was significantly associated with black and "other" race/ethnicity (as compared with whites), older age (65 years or older compared with under age 35), less education (high school graduate or less than high school education compared with college graduates), and no insurance coverage (compared with private, unmanaged). Higher service use was associated with Hispanic ethnicity, female gender, higher annual family income (over $\$ 20,000$ ), witnessing violence against an individual, screening positive for major depressive disorder, psychosis, or panic disorder, having more chronic health conditions than average, and perceiving a need for $\mathrm{ADM}$ services.

With regard to access to specialty ADM services, in the main effects model (not shown) higher HMO penetration rates and number of mental health and substance abuse facilities were significantly associated with increased utilization of specialty care. As in the model for access to any ADM services, we tested interactions between significant community-level vulnerability factors and insurance type (also statistically significant for this model $\left(F_{6,11,094}=4.871, p<.001\right)$. Only the interaction between HMO penetration 
and insurance type was significant $\left(F_{6,11,088}=2.524, p<.05\right)$, indicating that those with Medicare or Medicaid coverage were more likely to use specialty services in areas with higher HMO penetration. In the model with this interaction, lower specialty care use was associated with black race/ethnicity (as compared with whites), less education (high school graduate or less than high school education compared with college graduates), and having private, fully managed insurance coverage or no insurance (as compared with private, unmanaged). In contrast, higher service use was associated with screening positive for major depressive disorder, psychosis, or mania and perceiving a need for ADM services.

As the odds ratios for the interaction effects were not interpretable, to illustrate the effects of significant cross-level interaction between HMO penetration and insurance type we calculated odds ratios, confidence intervals, and significance levels for each insurance type at different values of HMO penetration equal to 20,30,40, and 50 percent (representing percentiles of the distribution at $18.8,45.3,63.3$, and 84.8 percent, respectively) for any specialty care (Table 3). These odd ratios indicate that for those with Medicare, Medicaid, private fully managed, and private partially managed insurance, the likelihood of utilization was higher in areas with higher HMO penetration. However, for those with other insurance or no insurance plan, the likelihood of utilization was lower in areas with higher HMO penetration. With the exception of Medicaid and other insurance plans, all insurance types at each level of HMO penetration had lower likelihoods of utilization when compared with private plans with no management. The likelihood of utilization was significantly lower, compared with private plans with no management, for those with Medicaid insurance living in areas with lower rates of HMO penetration. Likewise, the likelihood of utilization was significantly lower for those with private fully managed plans and those with no insurance at every rate of HMO penetration.

\section{CONCLUSIONS}

Our adaptation of Andersen and colleagues' Behavioral Model for Vulnerable Populations (Gelberg, Andersen, and Leake 2000) suggests that communitylevel enabling factors may be important for explaining use of ADM services, particularly for explaining specialty care use. Cross-level interactions between race/ethnicity, age, income level, and insurance type with community-level vulnerability factors were not significant, for access to any ADM care indi- 


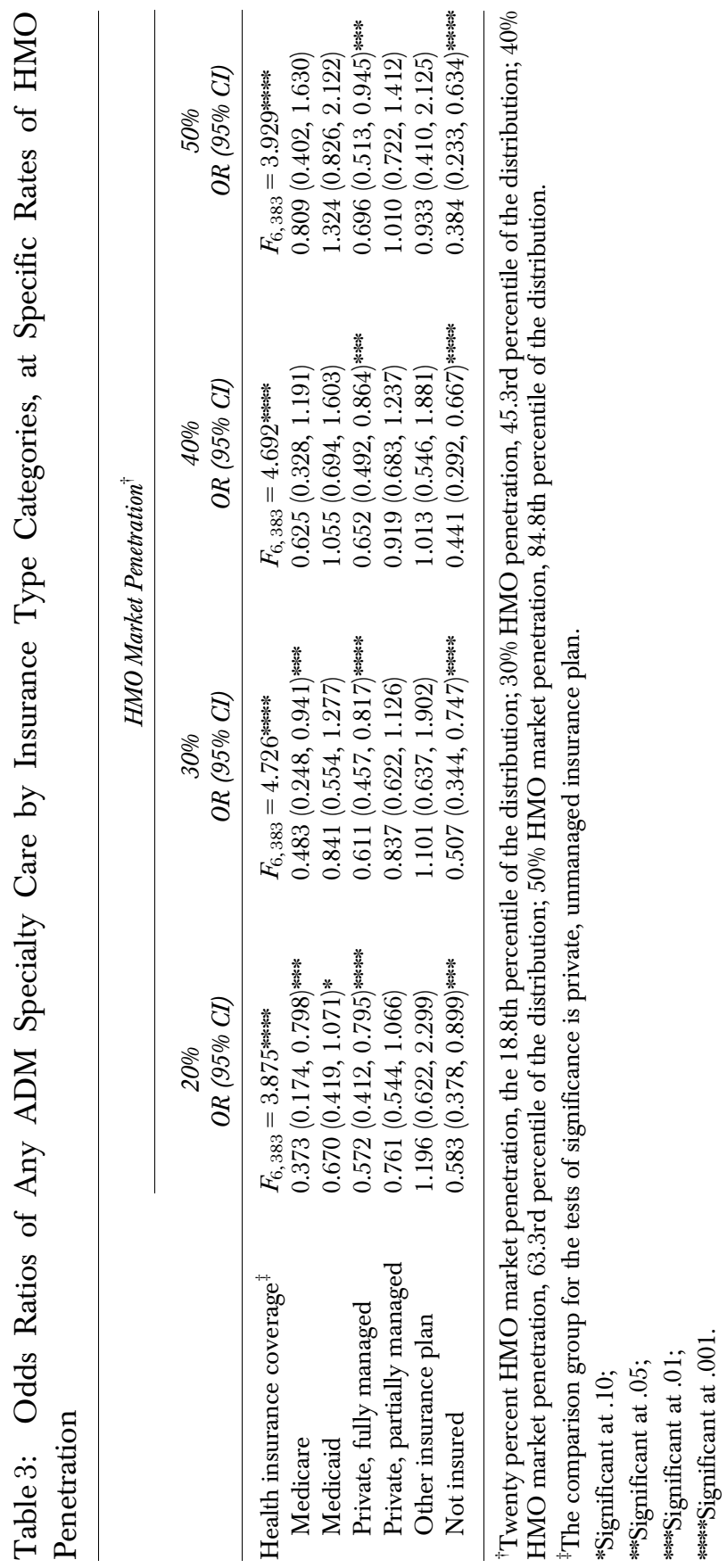


cating that vulnerable subgroups were not disproportionately affected by adverse health sector market conditions. Our results demonstrate that the likelihood of getting any ADM care decreases for some groups (blacks, Hispanics, "other" race/ethnicity, people age 65 or older, those with Medicaid coverage) when community characteristics are included in the model, but these decreases were small ( $<13$ percent change in odds ratio). Nevertheless, this finding demonstrates that health sector market factors and community socioeconomic disadvantage explain some of the disparities in service use by vulnerable subgroups.

Furthermore, the significant association of the number of inpatient and outpatient facilities with higher specialty care use, as well as the marginally significant association for psychiatrists per capita, suggest that communitylevel traditional enabling factors may be associated with better access to specialty care services. We also found that people with Medicare or Medicaid in areas with higher HMO penetration rates were more likely than those with private, unmanaged insurance to use specialty ADM care. This finding suggest that higher HMO market penetration is associated with better access for at least some people in the community (i.e., those with Medicare or Medicaid coverage), supporting the results of some previous studies (Sturm 1997; Goldman, McCulloch, and Sturm 1998; Grazier and Eselius 1998; Grazier et al. 1999; Gresenz, Stockdale, and Wells 2000; Hendryx et al. 2002).

Nevertheless, our finding that the likelihood of utilization is lower for the uninsured in areas with higher rates of HMO penetration lends some support to Rice's (2003) and others' contention that a greater presence of managed care may discourage service use for some vulnerable populations by shifting the burden of cost to the user. The uninsured are particularly vulnerable, as they must either pay for services out of pocket or rely on charity care. We did not examine whether HMO market penetration is associated with lower intensity/ volume of service use or lower quality of care, as some studies have suggested. In addition, we were not able to examine the relationship between communitylevel managed behavioral care "carve-outs" and access to specialty services, as no measure of this is currently available; however, the measure of HMO market penetration used here (obtained from the 2000 Area Resources File) is likely to be highly correlated with managed behavioral care "carve-outs."

Contrary to our expectations, we found that higher percent nonwhite population was significantly associated with higher access to any ADM care. Although the joint significance test for the interaction of race/ethnicity with percent population nonwhite was not significant below $p=.05$ (and thus not 
reported in our results), a significant effect for whites living in high nonwhite areas indicated that this group may be driving the significant effect found for percent nonwhite in the main effects model. This might suggest that, consistent with previous research, whites living in areas with primarily nonwhite populations may have experienced adverse personal circumstances due to illness, and may be more likely to use services because they are "sicker" than their nonwhite neighbors. Although we did not find support for this with our data, it is also possible that high service use by whites in these areas "crowds out" service use by nonwhites, particularly in areas with a lack of services. In addition, some of the literature on disparities in care has demonstrated how physician bias, uncertainty associated with presenting symptoms, and beliefs about nonwhite patients may operate in the clinical encounter to reduce both access to services and quality of care (Balsa and McGuire 2003; IOM 2003; Lutfey 2005; Lutfey and Freese 2005). Future research should investigate further whether cost-sharing and other cost-reduction strategies employed by managed care result in discrimination against nonwhites.

Finally, because the analyses employed cross-sectional data, we cannot completely rule out selection processes as an explanation for our results. For example, psychiatrists may chose to set up practices in areas where demand for services is high, thus creating the appearance that more psychiatrists per capita increases utilization when the opposite may in fact be true. Likewise, sicker individuals may move to certain areas where access to services are better, or they may be forced, due to adverse personal or financial circumstances, to move to socioeconomically disadvantaged neighborhoods where access to services tends to be more limited. Thus, the migration of such individuals to certain neighborhoods may actually be a cause, rather than a consequence, of seemingly better or worse access. More longitudinal research that links individual outcomes with the characteristics of their neighborhoods is necessary to sort out the causal direction of social context and ADM access outcomes.

Regardless, these findings highlight the importance of considering health sector market factors in understanding access to ADM services. Future studies should focus more directly on processes related to HMO market penetration, community socioeconomic disadvantage, and other health sector market factors, that are not uncovered in analyses carried out at this level of aggregation. In developing new policies and programs it will be important to understand better how these factors change, and whether intervening to change them shifts ADM utilization, either fundamentally (by lowering need) or by affecting migration patterns of providers and people at risk. 


\section{ACKNOWLEDGMENTS}

The authors would like to acknowledge and thank the following people for their contributions to the conceptualization of the analysis variables, technical assistance, and helpful comments: Ruth Klap, Susan Marquis, and Carole Gresenz. This research was supported by grants from the Robert Wood Johnson Foundation (038273) and the National Institute of Mental Health (P30MH068639:01).

Disclosures: No disclosures.

\section{NoTE}

1. A separate model for ADM specialty care with a random slope estimated at the county level indicated that the error variance for the county level was not significant; a similar model with a random slope for Census tract (nested within county) would not converge. The model with no random slopes did not produce substantively different results from the model presented in this paper.

\section{REFERENCES}

Andersen, R. M. 1995. "Revisiting the Behavioral Model and Access to Medical Care: Does It Matter?" Journal of Health and Social Behavior 36 (1): 1-10.

Balsa, A. I., and T. G. McGuire. 2003. "Prejudice, Clinical Uncertainty and Stereotyping as Sources of Health Disparities." Journal of Health Economics 22: 89-116.

Bell, R. 1999. Depression PORT Methods Workshop (I). Santa Monica, CA: RAND.

Berwick, D. M., J. M. Murphy, P. A. Goldman, J. E. Ware Jr., A. J. Barsky, and M. C. Weinstein. 1991. "Performance of a Five-Item Mental Health Screening Test." Medical Care 29 (2): 169-76.

Bryk, A. S., and S. W. Raudenbush. 1992. Hierarchical Linear Models. Newbury Park, CA: Sage Publications.

Bureau of Health Professions, U.S. Department of Health and Human Services. 1999 “Area Resource File."Available at http://bhpr.hrsa.gov/healthworkforce/data/ arf.htm

Bureau of Labor Statistics and Bureau of the Census. 2000. Avaliable at http:// www.bls.census.gov/cps/cpsmain.htm

Burnam, A., and A. S. Young. 1996. "Development of Healthcare for Communities Household Survey Instrument." UCLA/RAND Research Center on Managed Care for Psychiatric Disorders.

Chandra, A., and J. Skinner. 2003. "Geography and Racial Health Disparities." Working Paper 9513, National Bureau of Economic Research, Cambridge, MA. 
Chow, J. C. C., K. Jaffee, and L. Snowden. 2003. "Racial/Ethnic Disparities in the Use of Mental Health Services in Poverty Areas." American Journal of Public Health 93 (5): 792-7.

Cunningham, P.J. 1999. "Pressures on Safety Net Access: The Level of Managed Care Penetration and Uninsurance Rate in a Community." Health Services Research 34 (1): 255-65.

Cunningham, P. J., and P. Kemper. 1998. "Ability to Obtain Medical Care for the Uninsured: How Much Does It Vary across Communities?” Journal of the American Medical Association 280 (10): 921-7.

Feinson, M. C., and M. Popper. 1995. "Does Affordability Affect Mental Health Utilization? A United States-Israel Comparison of Older Adults." Social Science and Medicine 40 (5): 669-78.

Fiscella, K., and P. Franks. 2005. "Is Patient HMO Insurance or Physician HMO Participation Related to Racial Disparities in Primary Care?" American Journal of Managed Care 11 (6): 397-402.

Gelberg, L., R. M. Andersen, and B. D. Leake. 2000. "The Behavioral Model for Vulnerable Populations: Application to Medical Care and Outcomes for Homeless People." Health Services Research 34 (6): 1273-93.

Goldman, W., J. McCulloch, and R. Sturm. 1998. "Costs and Use of Mental Health Services before and after Managed Care." Health Affairs 17 (2): 40-52.

Grazier, K. L., and L. L. Eselius. 1998. "Mental Health Carve-Outs: Effects and Implications." Medical Care Research and Review 56 (s2): 33-59.

Grazier, K. L., L. L. Eselius, T. Hu, K. K. Shore, and W. A. G-Sell. 1999. "Effects of a Mental Health Carve-Out on Use, Costs, and Payers: A Four-Year Study." Journal of Behavioral Health Services and Research 26 (4): 381-9.

Grembowski, D. E., D. Martin, D. L. Patrick, P. Diehr, W. Katon, B. Williams, R. Engelberg, L. Novak, D. Dickstein, R. Deyo, and H. I. Goldberg. 2002. "Managed Care, Access to Mental Health Specialists, and Outcomes among Primary Care Patients with Depressive Symptoms." Journal of General Internal Medicine 17: 258-69.

Gresenz, C. R., S. E. Stockdale, and K. B. Wells. 2000. "Community Effects on Access to Behavioral Health Care." Health Services Research 35 (1): 293-307.

Hendryx, M. S., and M. M. Ahern. 2001. "Access to Mental Health Services and Health Sector Social Capital." Administration and Policy in Mental Health 28 (3): 205-18.

Hendryx, M. S., M. M. Ahern, N. P. Lovrich, and A. H. McCurdy. 2002. "Access to Health Care and Community Social Capital.” Health Services Research 37 (1): 87103.

Hendryx, M. S., M. E. Urdaneta, and T. Borders. 1995. "The Relationship between Supply and Hospitalization Rates for Mental Illness and Substance Use Disorders." Journal of Health Administration 22 (2): 167-76.

Institute of Medicine. 2003. A Shared Destiny: Community Effects of Uninsurance. Washington, DC: The National Academies Press.

Karlin, B. E., and M. P. Norris. 2005. "Public Mental Health Care Utilization by Older Adults." Administration and Policy in Mental Health Services Research 12: 1-7, epub. 
Kessler, R. C., G. Andrews, D. Mroczek, B. Ustun, and H. U. Wittchen. 1998. "The World Health Organization Composite International Diagnostic Interview Short-Form (CIDI-SF)." International Journal of Methods in Psychiatric Research 7 (4): 171-85.

Krause, N. 1999. "Mental Disorder in Late Life: Exploring the Influence of Stress and Socioeconomic Status." In Handbook of the Sociology of Mental Health, edited by C. S. Aneshensel and J. C. Phelan, pp. 183-208. New York: Kluwer Academic/ Plenum Publishers.

Litaker, D., and R. D. Cebul. 2003. "Managed Care Penetration, Insurance, Status, and Access to Health Care." Medical Care 41 (9): 1086-95.

Little, R. J. 1988. "Missing Data Adjustments in Large Surveys." Journal of Business and Economic Statistics 6: 287-301.

Lutfey, K. 2005. "On Practices of 'Good Doctoring': Reconsidering the Relationship between Provider Roles and Patient Adherence.” Sociology of Health and Illness 27 (4): 421-47.

Lutfey, K., and J. Freese. 2005. "Toward Some Fundamentals of Fundamental Causality: Socioeconomic Status and Health in the Routine Clinic Visit for Diabetes." American Journal of Sociology 110 (5): 1326-72.

Milliken, G. A., and F. A. Graybill. 1970. "Extensions of the General Linear Hypothesis Model." Journal of the American Statistical Association 65 (330): 797-807.

Pfeffermann, D., and L. LaVange. 1989. "Regression Models for Stratified Multi-Stage Cluster Samples.” In Analysis of Complex Surveys, edited by C. J. Skinner, D. Holt, and T. M. F. Smith. New York: John Wiley \& Sons.

Rasbash, J., F. Steele, and W. Browne. 2003. A User's Guide to MLwiN. London: Centre for Multilevel Modeling.

Rice, T. 2003. "The Impact of Const Containment Efforts on Racial and Ethnic Disparities in Healthcare: A Conceptualization.” In Unequal Treatment: Confronting Racial and Ethnic Disparities in Healthcare, edited by B. D. Smedley, A. Y. Stith, and A. R. Nelson, pp. 699-721. Washington, DC: Institute of Medicine, The National Academies Press.

Rubin, D. B. 1987. Multiple Imputation for Non-Response in Surveys. New York: John Wiley \& Sons.

Sherbourne, C. D., and A. L. Stewart. 1991. "The MOS Social Support Survey." Social Science and Medicine 32: 705-14.

Sturm, R. 1997. "How Expensive Is Unlimited Mental Health Care Coverage under Managed Care?" Journal of the American Medical Association 278 (18): 1533-37.

Sturm, R., C. Gresenz, C. Sherbourne, K. Minnium, R. Klap, J. Bhattacharya, D. Farley, A. S. Young, M. A. Burnham, and K. B. Wells. 1999. "The Design of Health Care for Communities: A Study of Health Care Delivery for Alcohol, Drug Abuse, and Mental Health Conditions." Inquiry 36: 221-33.

Thorpe, K. E., E. E. Seiber, and C. S. Florence. 2001. "The Impact of HMOs on Hospital-Based Uncompensated Care." Journal of Health Politics and Policy Law 26 (3): 543-55.

U.S. Census 2000 Summary File 3-Sample Data. Table P87. [Last accessed on November 18, 2004]. Available at http://factfinder.census.gov 
U.S. Census 2000 Summary File 1. Table P8. [Last accessed on April 26, 2005]. Available at http://factfinder.census.gov

U.S. Census Bureau and U.S. Department of Commerce. 1999. ZIP Code Business Patterns Data (CD-ROM).

Virnig, B., Z. Huang, N. Lurie, D. Musgrave, A. M. McBean, and B. Dowd. 2004. "Does Medicare Managed Care Provide Equal Treatment for Mental Illness across Races?” Archives of General Psychiatry 61: 201-5.

Weissman, E., K. Pettigrew, S. Sotsky, and D. A. Regier. 2000. "The Cost of Access to Mental Health Services in Managed Care.” Psychiatric Services 51 (5): 664-6.

Wilk, J. E., J. C. West, W. E. Narrow, D. S. Rae, and D. A. Regier. 2005. "Access to Psychiatrists in the Public Sector in Managed Health Plans." Psychiatric Services 56 (4): 408-10.

World Health Organization (WHO). 1990. Composite International Diagnostic Interview (CIDI) (Version 1.0). Geneva: World Health Organization. . 1992. The Alcohol Use Disorders Identification Test (AUDIT). Guidelines for Use in Primary Health Care. Geneva: World Health Organization.

1997. Composite International Diagnostic Interview, Core Version 2.1 Computer Manual. Geneva: World Health Organization. 